\title{
Experimental and Numerical Investigation of the Flow Topology During Airdrop Operations
}

\author{
Sven Geisbauer, Niko Schade* and Stephan Enk* \\ DLR Institute of Aerodynamics and Flow Technology, 38108 Braunschweig, Germany \\ Hauke Schmidt* and Jürgen Arnold* \\ DLR Institute of Aeroelasticity, 37073 Göttingen, Germany
}

This paper reviews experimental and numerical simulations of the dropping of simplified cargo supplies from a generic military transport aircraft carried out at DLR. Aiming at developing a well-validated process chain to accurately compute the trajectories and attitudes of airdropped cargo supplies in the proximate vicinity of the aircraft, extensive low-speed wind tunnel tests were conducted as a means to validate the numerical simulation. A simulation environment was established, coupling unsteady Navier-Stokes CFD methods with multi-body simulation methods, allowing for the precise computation of the time-dependent aerodynamic and flight mechanic behaviour of an airdropped supply. A comparison of selected results between the numerically simulated airdrop and the wind tunnel data is presented.

\section{Nomenclature}

$\begin{array}{llll}\alpha & \text { Aircraft angle of attack } & u & \text { Velocity component in x-direction } \\ \Delta t & \text { Time interval } & u_{\infty} & \text { Onflow velocity component in x-direction } \\ \Delta x & \text { Translation in x-direction } & v & \text { Velocity } \\ \Delta y & \text { Deviation in y-direction } & v_{\infty} & \text { Onflow velocity } \\ \Delta \Theta & \text { Pitch angle variation } & v_{x}, v_{y}, v_{z} & \text { Velocities in } x \text {-, } y \text {-, }, z \text {-direction } \\ \rho & \text { Density } & v_{x}^{*}, v_{y}^{*}, v_{z}^{*} & \text { Relative velocities in } x \text {-, } y \text {-, } z \text {-direction } \\ \Phi, \Theta, \Psi & \text { Roll, pitch, yaw angles } & & \text { (related to } v_{\infty} \text { ) } \\ \Phi^{*}, \Theta^{*}, \Psi^{*} & \text { Relative roll, pitch, yaw angles } & W & \text { Width } \\ & \text { (related to } \alpha) & x_{b}, y_{b}, z_{b} & \text { Body-fixed coordinates } \\ F_{x} & \text { Force in x-direction } & y^{+} & \text {Non-dimensional wall distance } \\ g & \text { Acceleration due to gravity } & \text { CAD } & \text { Computer Aided Design } \\ H & \text { Height } & C^{2} A^{2} S^{2} E & \text { Center for Computer Applications in } \\ J_{y y} & \text { Moment of inertia about y-axis } & & \text { Aerospace Science and Engineering } \\ L & \text { Length } & \text { CFD } & \text { Computational Fluid Dynamics } \\ m & \text { Mass } & \text { CFRP } & \text { Carbon Fibre Reinforced Plastics } \\ M & \text { Model scale, moment } & \text { CPU } & \text { Central processing unit } \\ M_{y} & \text { Moment about y-axis } & \text { D } & \text { Dimensional } \\ n & \text { Number of sampling points } & \text { DES } & \text { Detached-eddy Simulation } \\ p, q, r & \text { Roll, pitch, yaw rates } & \text { DNW } & \text { German-Dutch Wind Tunnels } \\ t & \text { Time } & \text { DLR } & \text { German Aerospace Center } \\ t^{*} & \text { Time step size } & \text { DOF } & \text { Degree of freedom }\end{array}$

${ }^{*}$ Research scientist 


$\begin{array}{llll}\text { fps } & \text { Frames per second } & \text { NWB } & \text { Low-speed Wind Tunnel Braunschweig } \\ \text { HDG } & \text { Hole definition geometry } & \text { PIV } & \text { Particle image velocimetry } \\ \text { MBS } & \text { Multi-body Simulation } & \text { RANS } & \text { Reynolds-averaged Navier-Stokes (equations) } \\ \text { MPM } & \text { Model Positioning Mechanism } & \text { R/C } & \text { Radio-controlled }\end{array}$

\section{Introduction}

Ardrop operations of cargo pallets and humanitarian aid supplies from military transport aircraft are Acarried out in very special aerodynamic and flight mechanic circumstances. During airdrop operations the aircraft often operates in the low-speed regime at low altitudes with deployed high-lift devices, which increase the vorticity and the downwash in the flow field. Additionally, the unique geometric shape of modern military transport aircraft, in particular the voluminous fairings of the landing gears on the one hand and the high upsweep angle of the rear fuselage on the other hand significantly contribute to a highly vortical flow in the rear section of the aircraft and farther downstream. Flying with lowered cargo ramp even aggravates this situation. ${ }^{1}$

In the first phase of the dropping sequence, beginning with the cargo release until it leaves the area of influence of the aircraft, the complex flow field behind the aircraft interacts with the dropped supply. Especially very lightweight and aerodynamically sensitive supplies are prone to colliding with the aircraft structure and, thus, pose a significant threat to flight safety. ${ }^{2,3}$ Furthermore, at low altitudes, the change of the overall centre of gravity generally poses a significant risk potential to the crew, the cargo and the aircraft. Therefore, the ability to accurately predict the trajectory and time-dependent attitude of an airdropped supply as well as the resulting motion of the aircraft is of great importance.

Traditionally, separation investigations relied on wind tunnel campaigns and flight tests. Apart from the high risk potential of flight tests, the cost factor and often time-consuming certification procedure led to an increasing demand to develop accurate and well-validated simulation tools. Within the international FourPowers Air Senior National Representative Long Term Technology Projects a technical group was established to improve the precision of airdrop operations by improving the dropping sequence itself and by fostering the development of airdrop simulation tools. ${ }^{3,4,5}$

Past simulation tools were based on low-fidelity methods. The trajectory prediction was mostly carried out in 2D with a 3-DOF motion. The aerodynamic data was based on inviscid computations or aerodynamic lookup tables. ${ }^{3,6}$ In order to increase the precision of modern airdrop systems, more accurate simulation tools allowing for three-dimensional, 6-DOF motion need to be applied. In addition, a thorough understanding of the flow field around the aircraft and the supply is mandatory to assess interference effects and their influence on airdrop precision.

In recent years the advance in the application of numerical methods allowed for a more accurate and time-dependent simulation of airdrop operations. Extensive experimental test campaigns were carried out, focussing on precisely assessing the flow field as well as the acting forces during airdrop operations, mostly in order to provide validation data. ${ }^{1,7,8,9}$ Nowadays, simulation methods are in use for a wide variety of separation problems, like the cover separation of a missile, the store separation from an aircraft or paratrooper deployment. ${ }^{10,11,12,13}$ Moreover, the simulation of the flow field about cargo containers and parachutes as well as determining the acting aerodynamic forces has been pushed into the spotlight. ${ }^{7,14,15}$

Combining flight mechanics with state-of-the art CFD methods, like those based on solving the Reynoldsaveraged Navier-Stokes (RANS) equations and Detached-Eddy Simulation (DES), into a coupled simulation environment represents the highest fidelity level currently available for airdrop simulations. ${ }^{16}$ These simulations allow for precisely analyzing the airflow interference effects as well as the acting loads during deployment and, thus, may contribute to a partial replacement of flight tests for certification purposes.

The present paper gives an overview on airdrop experiments carried out in the DNW-NWB low speed wind tunnel facility in Braunschweig, Germany. ${ }^{17}$ Generic cargo supplies with gradually increased geometric complexity and varying masses were dropped from a wind tunnel model of a generic transport aircraft with open ramp. The data was used for the validation of the numerical simulation. 
Aiming at accurately simulating the first phase of an airdrop sequence, beginning from cargo release until it leaves the area of influence of the aircraft, a parameter study to capture the relevant flow features was conducted in advance with the DLR TAU RANS code. ${ }^{18}$ These simulations were validated using data from PIV measurements. ${ }^{1}$

In a next step a process chain based on the DLR TAU code and the multi-body simulation software SIMPACK ${ }^{19}$ was established. It allows for the non-linear coupled simulation of the aerodynamics and the flight mechanics of two or more bodies in relative motion. The coupling of both tools accounts for an accurate computation of the aerodynamic forces and moments acting on the supply and the resulting motion in the gravity field.

\section{Experimental Airdrop Investigation}

Wind tunnel airdrop experiments were carried out within four campaigns between 2006 and 2008, using a generic military transport aircraft configuration with lowered ramp. Geometrically simplified, cuboid cargo models with and without parachute model were dropped from the carrier aircraft. The main objective was to provide reliable data for the validation of the numerical airdrop simulation. The repeatability of the experiments was high, even for very lightweight and, thus, aerodynamically sensitive cargo bodies, which proved the suitability for the validation of the numerical simulation.

\section{II.A. Wind Tunnel Scaling Requirements}

To draw conclusions from wind tunnel results to the behaviour of the full scale object several similarity parameters like the Reynolds and Mach numbers need to be in good agreement. When comparing the trajectories of two bodies dropped from an aircraft, once in wind tunnel scale and once in full scale, it becomes clear that both bodies are falling the same absolute distance within a given time interval $\Delta t$ due to gravity. The relative falling distance referred to the respective characteristic length, however, is different. Therefore, to guarantee the comparability of the trajectories it has to be ensured that the relative falling distances are equal within a given time interval $\Delta t$. For a point mass this is achieved by reducing the ramp velocity $v_{M}$ based on the model scale $M$

$$
v_{M}=\sqrt{M} \cdot v_{O}
$$

compared to the full scale ramp velocity $v_{O}$. Consequently, time will also scale according to Eq. (2).

$$
t_{M}=\sqrt{M} \cdot t_{O}
$$

In case of voluminous bodies, the comparability of the trajectories is achieved if the Froude numbers, i. e. the ratio between forces due to inertia to those due to gravity, are equal. Finally, Eq. (3) gives a relation between the densities of two bodies, their masses and the model scale.

$$
\frac{\rho_{M}}{\rho_{O}} \cdot S^{3}=\frac{m_{M}}{m_{O}}
$$

For a deeper insight into the derivation of the scaling requirements of a dropped body the reader is referred to Refs. 20 and 21.

\section{II.B. Experimental Setup}

Cuboid cargo models were used in the different wind tunnel campaigns, being manufactured of different materials and, thus, allowing for a variation of the mass per unit area. The homogeneously distributed masses of these bodies range from $60 \mathrm{~g}$ up to $550 \mathrm{~g}$, representing realistic masses ranging from $500 \mathrm{~kg}$ to $5000 \mathrm{~kg}$ when converted back into full scale according to Eq. (3). The canopy of the parachute was modeled as a rigid, unslotted hemisphere, containing a vent hole on its symmetry axis. The inflation process was not taken into account. The experimental setup represents a drogue extraction with fully inflated drogue parachute and stretched extraction lines. The modular manufacturing allowed for two different ways of the canopy model being attached to the cargo body, either rigidly or by means of a 1-DOF joint. 


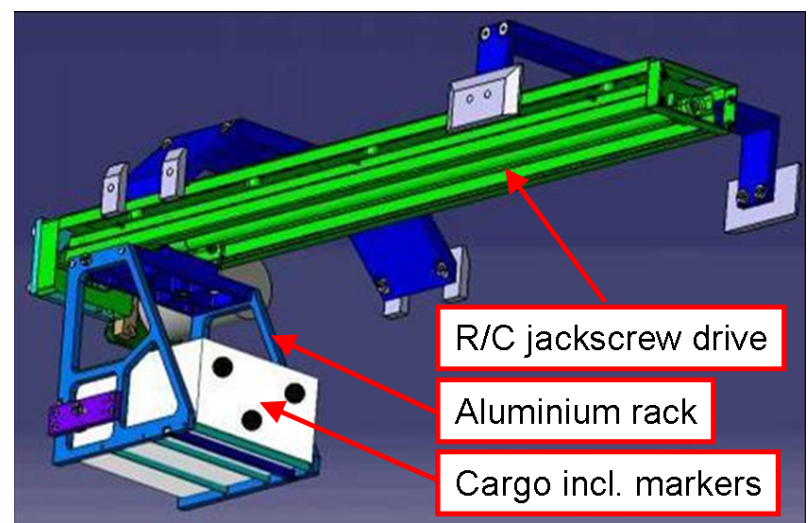

(a) CAD model of the ejection mechanism integrated into the cargo compartment.

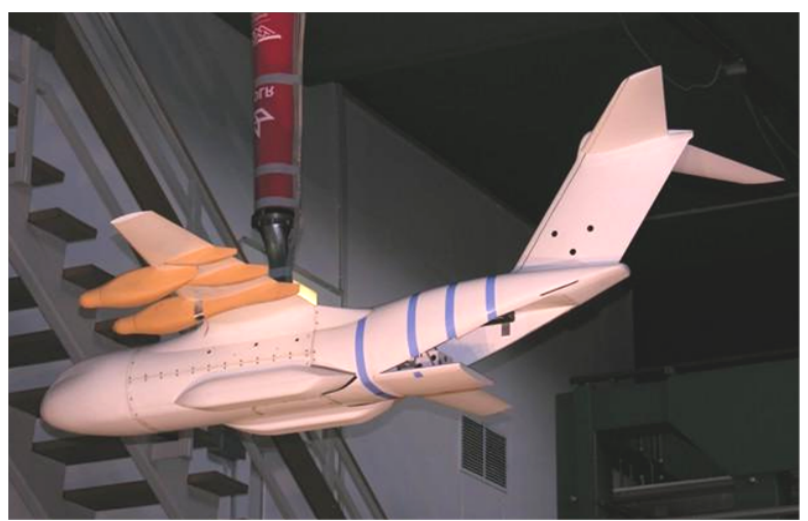

(b) Wind tunnel airdrop setup using a generic transport aircraft with lowered ramp.

Figure 1. Experimental setup for the airdrop investigations.

The validation data presented in this paper was obtained from experiments with a cargo body manufactured of lime wood. The parachute was rigidly attached to the cargo by means of three bolted, circular CFRP-rods, sized $3 \mathrm{~mm}$ in diameter and $215 \mathrm{~mm}$ in length. An evaluation of the wind tunnel airdrop campaigns with different cuboid bodies of varying complexity will be published in $2011 .{ }^{22}$

The DNW-NWB wind tunnel in Braunschweig is focussed on industrial applications and can be operated with either open, closed or slotted wall test section. The test section offers a cross section of $3.25 \mathrm{~m} \mathrm{x} 2.8 \mathrm{~m}$ and a length of $6 \mathrm{~m}$. Regarding the onflow velocity for the open test section, the operating range lies between $0 \leq v_{\infty} \leq 70 \mathrm{~m} / \mathrm{s}$. The experimental tests were carried out in the lower velocity regime.

For the ejection of the cargo with a initial velocity derived from real operations in conjunction with Eq. (1) an ejection mechanism was integrated into the cargo compartment of the aircraft as depicted in figure 1(a). It consists of a support rack made of aluminium, accelerating the cargo through the compartment, which is mounted to a radio-controlled jackscrew drive. As soon as the driven rack reaches its rearmost position, just above the edge of the cargo ramp, the cargo body separates due to its inertia. The position and attitude of the cargo in this particular moment exhibit the initial conditions for the numerical simulation. For the experiments with attached parachute the mechanism was slightly modified with an additional arrestor plate, preventing the model of prematurely tilting.

To allow for an undisturbed flow around the aircraft in the area of interest, the aircraft was supported by a dorsal sting to the Model Positioning Mechanism (MPM) of the DNW-NWB. ${ }^{17}, 23$ The collector of the test section was protected by a net to catch the dropped bodies. Figure 1(b) gives an overview on the wind tunnel setup. Figure 2 gives an overview on two of the cargo bodies used during experiment. More details on the physical properties of the test body are given in table 1 .

Table 1. Details on the experimental airdrop body.

\begin{tabular}{lc}
\hline \hline Material & Lime wood \\
\hline Dimensions cargo body $(L \times H \times W)$ & $110 \mathrm{~mm} \times 50 \mathrm{~mm} \times 90 \mathrm{~mm}$ \\
Length CFRP rods & $215 \mathrm{~mm}$ \\
Diameter of each CFRP rod & $3 \mathrm{~mm}$ \\
Outer diameter parachute & $130 \mathrm{~mm}$ \\
Vent hole diameter & $20 \mathrm{~mm}$ \\
\hline Mass cargo body & $335 \mathrm{~g}$ \\
Mass CFRP rods & $3 \cdot 4 \mathrm{~g}$ \\
Mass canopy & $22 \mathrm{~g}$ \\
Overall mass of test body & $369 \mathrm{~g}$ \\
\hline \hline
\end{tabular}




\section{II.C. Measurement Instrumentation}

An optical tracking method consisting of two cameras was applied to evaluate the trajectories and attitudes of the dropped bodies. The video cameras, type Mikrotron MC 1310 CMOS, working with a resolution of $1280 \times 1024$ pixels and a frame rate of $300 \mathrm{fps}$ were positioned at a viewing angle of approx. $60^{\circ}$ apart from each other. To track the moving bodies, each of them was equipped with three white, circular markers on their side faces as shown in figures 1 (a) and 2. The cameras were calibrated by evenly distributing up to 16 light sources with known position in the trajectory space. It allows for the determination of position and attitude of the dropped body by computationally evaluating two corresponding marker images with the software picColor. ${ }^{24}$ Based on the spatial position of each of the three markers the position of the centre of gravity of the body as well as the attitude of the body were determined. The rate of change of position and attitude within a given time interval allows for derivating the translational and rotational velocities and accelerations, respectively.

The accuracy of the calibrated setup was checked by using rotated cuboids in fixed positions both inside and outside of the calibration space. Within the calibration space the spatial deviations in tracking these test bodies proved to not exceed $1 \mathrm{~mm}$, which is less then $1 \%$ of the cuboid's length. For highly rotated cuboids positioned outside of the calibration space, the maximum lateral displacement amounts to $\Delta y \approx 4 \mathrm{~mm}$. The

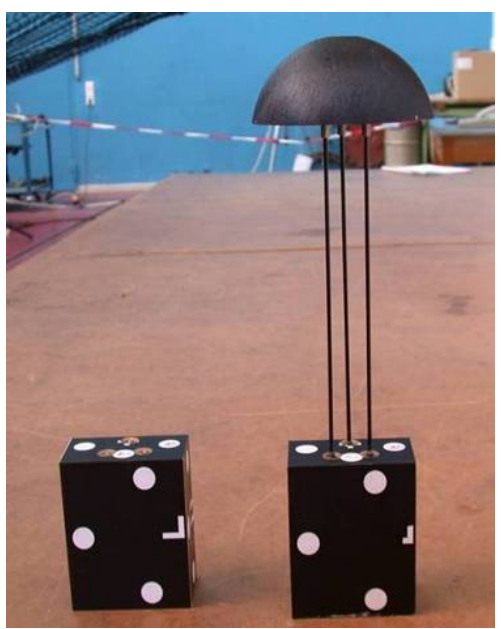

Figure 2. Overview on different experimental cargo bodies. The right body's CFRP rods have a length of $465 \mathrm{~mm}$. A similar body with a rod length of $215 \mathrm{~mm}$ was also used in experiment and chosen for the validation of the numerical simulation. achieved accuracy was found to be sufficient for the validation of the numerical simulation.

\section{Numerical Airdrop Investigation}

\section{III.A. Computational Fluid Dynamics Solver TAU}

The computation of the flow field about the aircraft and the cargo was carried out with the DLR TAU-code, a Reynolds-averaged Navier-Stokes solver which is under continuous development by DLR. ${ }^{18,25,26}$ The main developments of the TAU-code originated from the German CFD project MEGAFLOW, consolidating developments of DLR, aircraft industry and universities. TAU is an unstructured, edge-based solver in cell-vertex or cell-centered formulation, making use of the advantages of hybrid grids for the resolution of viscous shear layers near walls. A dual-grid approach is used to allow for flow computations on grids being independent of the cell types of the initial grid. TAU solves the compressible, three-dimensional, time-accurate Reynoldsaveraged Navier-Stokes equations. The spatial discretization with either upwind or central schemes is based on a finite volume formulation. The temporal discretization may either be carried out with an explicit Runge-Kutta scheme or with an implicit Lower-Upper Symmetric Gauss-Seidel (LU-SGS) scheme. Several convergence acceleration techniques like residual smoothing or multigrid schemes may be applied. Different turbulence models of varying fidelity, ranging from standard one-equation models to Reynolds stress models, have been implemented in TAU. The standard turbulence model is the one-equation Spalart-Allmaras model. ${ }^{27}$ Today, TAU is routinely used in the European aeronautical industry for external aerodynamics.

Before starting with the unsteady airdrop simulation, a time-accurate initial solution based on a wellconverged steady solution was computed. The flow solver was employed in cell-vertex mode, the spatial discretization was carried out using a central scheme with artificial matrix dissipation. ${ }^{28,29,30}$ An implicit Backward-Euler scheme with linear LU-SGS solver was applied for the temporal discretization. The timeaccurate computation was conducted in employing a dual time stepping scheme. Preceding numerical studies addressed the influence of the turbulence model to the flow solution behind the aircraft. Negligible difference was found between one- and two-equation turbulence model. It was therefore decided to conduct all subsequent computations with the Spalart-Allmaras turbulence model with modification by Edwards. ${ }^{27,31}$ 
The grids used for the simulations presented in this paper were created with the hybrid grid generator Centaur, a commercial software developed by CentaurSoft. ${ }^{32}$ Separate grids were generated for the aircraft and the cargo with rigidly attached parachute, each of them such to yield a non-dimensional first wall distance of $y^{+} \leq 1$. In order to use symmetric full model meshes, the aircraft was gridded as half-model and then mirrored along its symmetry plane. The grid is refined in the trajectory space behind and below the aircraft to accurately resolve the predominant flow features along the trajectory. The final full model grid contained $24.6 \cdot 10^{6}$ points. The cargo-parachute model was meshed as quarter-model and then mirrored twice. In contrast to the experimental setup the three CFRP-rods were neglected in CFD. The final full model mesh of the cargo-parachute model contains $2.7 \cdot 10^{6}$ points. Its surface mesh is depicted in figure $3(\mathrm{a})$. The final computational grid was created making use of the Chimera or overset grid technique. It contains $27.3 \cdot 10^{6}$ points. Figure 3(b) shows the assembled final grid as well as the refined trajectory space beneath the aircraft.

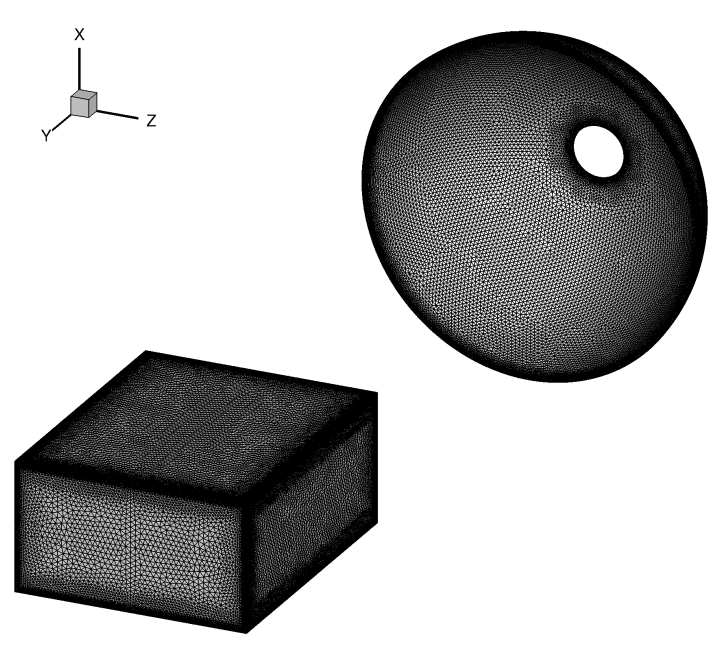

(a) Surface mesh of the airdrop body used in the numerical simulation.

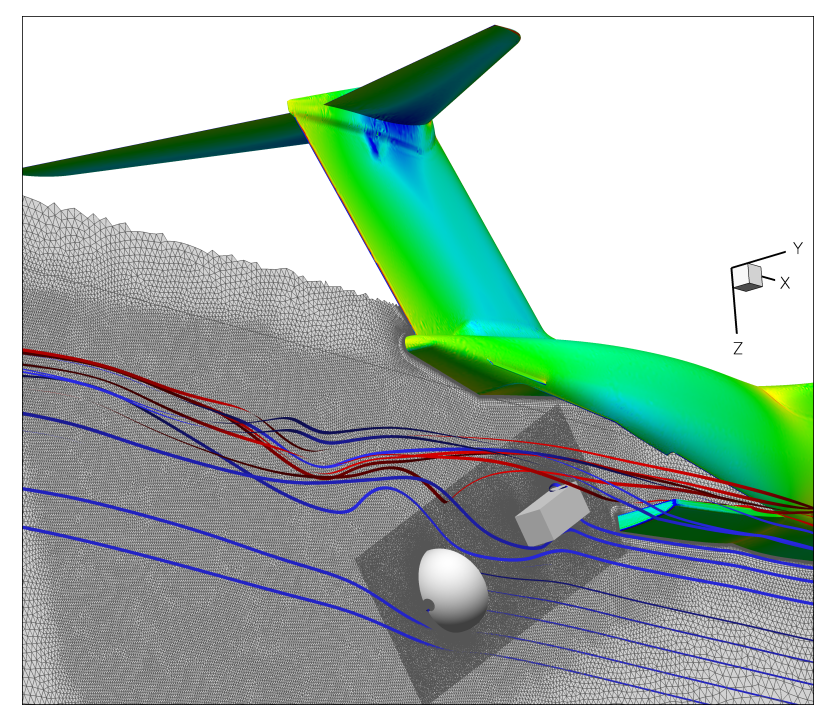

(b) Overset grid setup for the numerical simulation and vortical flow interactions behind the aircraft. Aircraft colouring by pressure.

Figure 3. Overview on grid setup and cargo model for the airdrop simulation.

\section{III.A.1. Chimera Approach}

The Chimera or overset grid technique provides the capability of performing computations with overlapping meshes. Avoiding a remeshing or grid deformation during the simulation the Chimera approach offers a convenient way to simulate manoeuvring aircraft with moving control surfaces or, as applied here, any kind of separation problem. A deeper insight into the Chimera functionality of the DLR TAU code is given in Refs. 33 and 34. An application of the technique for a manoeuvring aircraft is given in Ref. 35. The current implementation in the TAU code handles multi-body simulations with either predefined mesh overlap regions or by employing a semi-automatic hole-cutting process. The latter is the preferred approach for applications in which the trajectory of a moving body is unknown in advance, like in airdrop simulations.

To apply this technique the user has to define a so-called hole definition geometry (HDG) for each moving body. The HDG is connected to the respective body and moves along with it, cutting a hole along the HDG boundary faces into the background meshes and, thus, ensuring a valid mesh topology during the simulation. The Chimera approach can be applied in both sequential and parallel mode. The search algorithm is based on an alternating digital tree methodology.

\section{III.A.2. TAU Motion Module}

The TAU motion module is part of the TAU code environment and provides a library of functions for the simulation of arbitrary rigid-body motions. The module relies on a hierarchical order of the moving bodies 
in such a way that, for example, an aileron inherits the motion of the wing or an elevator the motion of the horizontal tail plane. The motion module plays a vital role in the coupled simulation process between TAU and SIMPACK. It interprets the resulting motion data from SIMPACK and initiates the relative grid displacement with the help of the Chimera technique.

\section{III.A.3. Influence of Turbulence Modeling}

In order to assess the influence of applying the RANS approach to the highly vortical flow field around the aircraft, preceding studies had been carried out in which RANS results and results from Detached-eddy Simulations were compared to experimental data. The latter was obtained by means of the particle image velocimetry (PIV). A delayed DES (DDES) ${ }^{36}$ was chosen for this study to avoid grid induced separations. In figure 4(a) the mean velocity components in streamwise direction of DES, experiment and RANS are compared in a $x=$ const. slice behind the open ramp. RANS and DES correlate well with the experiment. In addition, the DES resolves the sponson vortices. Having a look at the vertical velocity component in figure 4(b) the RANS results better match the experimental data whereas the DES underestimates the size of the region with high vertical velocities.

As exemplarily shown here the preceding studies proved the general feasibility of DES. However, the DES results were not found to be of higher quality than the RANS results for this particular test case. The advantage of the DES here consists in the capability to resolve unsteady flow phenomena, e. g. like frequency spectra. On the other hand the computational efforts in applying a DES significantly exceed those of a RANS simulation. Therefore, it was decided to focus on RANS methods only for the successive work.

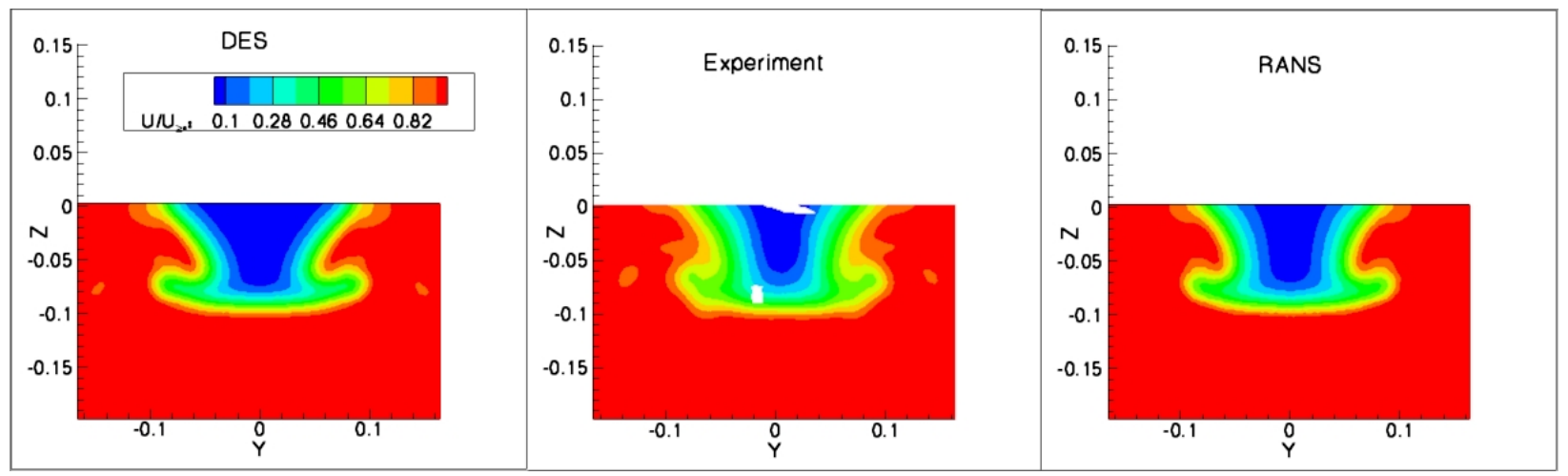

(a) Streamwise velocity component in a slice behind the open ramp of the aircraft.

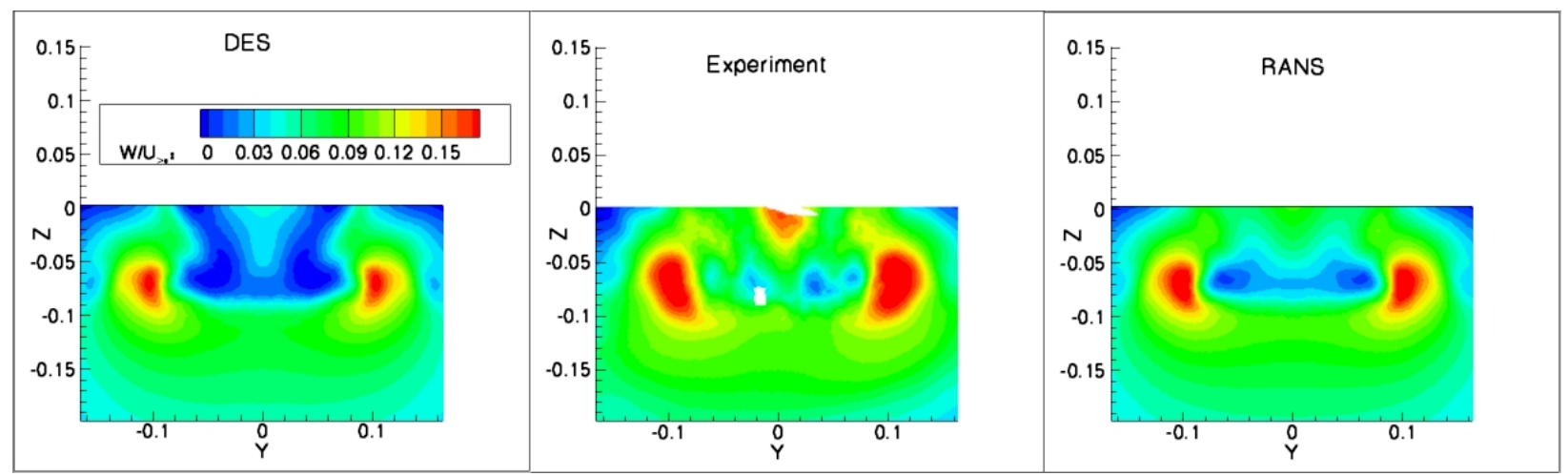

(b) Vertical velocity component in a slice behind the open ramp of the aircraft.

Figure 4. Comparison of results from DES, experiment and RANS methods. 


\section{III.B. Multi-Body Simulation Software SIMPACK}

For the solution of the flight mechanical equations of motion the commercial multi-body system (MBS) software SIMPACK was used. ${ }^{19}$ SIMPACK is based on a DLR development and represents a standard engineering tool in the automotive and railway design. The functionality in terms of aeronautical engineering enables the simulation of complex dynamical systems as well as individual motion tracking, for instance with Euler angles. For more information on SIMPACK the reader is referred to Ref. 37.

Within the present work the MBS model in SIMPACK comprises two bodies, the aircraft and the cargo with attached parachute, shown in figure 5. The aircraft was modeled as a rigid, non-moving body whereas the cargo-parachute body was modeled via a 6-DOF joint, allowing for an arbitrary spatial rigid-body motion. The translational motion of the moving body has been described in the parent coordinate systems whereas the rotational motion has been described in body-fixed coordinates.

To avoid singularities associated with the Euler angle formulation the rigid body motion has been converted to a quaternion formulation in an intermediate step. A deeper insight into the quaternion formulation is given in Ref. 38.

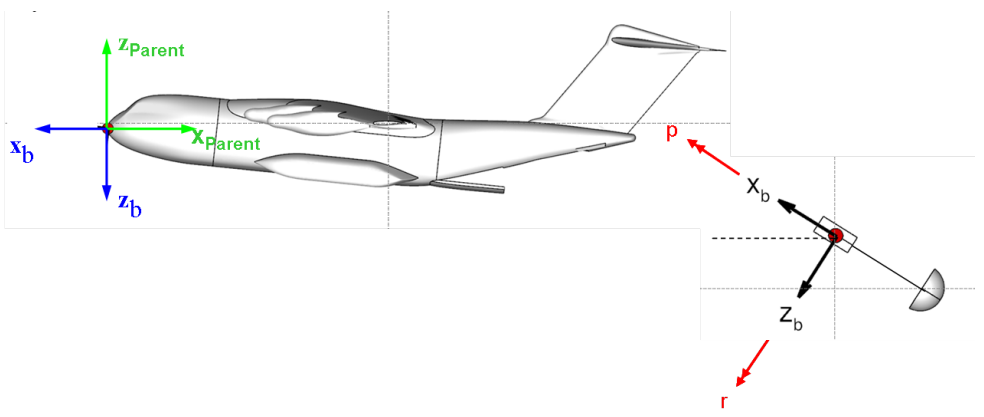

Figure 5. Overview on the bodies used in SIMPACK. The origin of the respective local coordinate system is indicated by a red dot.

\section{III.C. Coupled TAU-SIMPACK Simulation Environment and Verification}

The coupled process chain is based on a classical co-simulation approach using two programs which exchange data within a given time step. Herewith, TAU provides the aerodynamic forces and moments acting on both aircraft and cargo. The data is exchanged via a TCP/IP socket to SIMPACK and used to compute the resulting motion parameters for the next time step. In particular, these comprise the rotational and translational displacement of the body as well as the respective velocities. The data is transferred back to the TAU motion module, yielding the new position and attitude of the cargo for the next time step. Figure 6 schematically depicts the setup of the coupled TAU-SIMPACK simulation environment. As the temporal coupling scheme is of first-order accuracy there is the need of employing relatively small time step sizes for an appropriate numerical accuracy, especially for high rotational or translational velocities. According to the temporal discretization in the experiments (300 fps) the unsteady coupled simulation was conducted with a sampling frequency of $300 \mathrm{~Hz}$. A detailed description of the simulation environment is given in Ref. 39 .

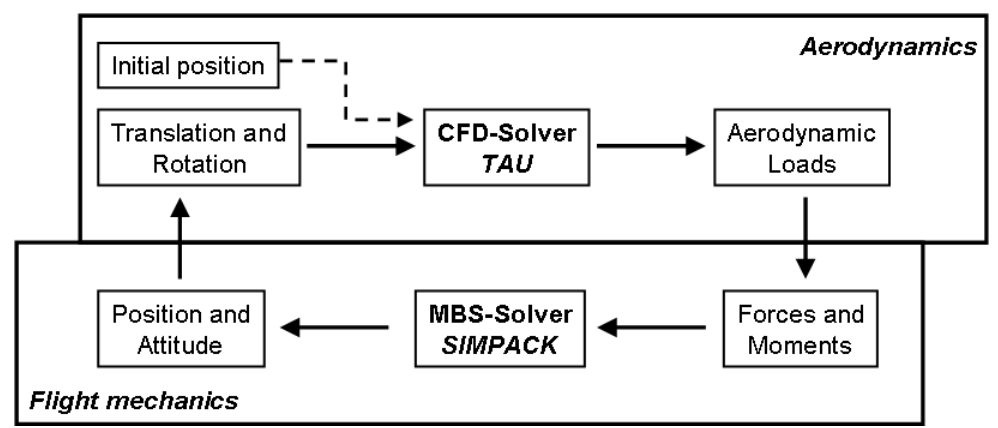

Figure 6. Schematic overview on the coupled simulation environment. 


\section{III.C.1. Verification of Process Chain}

The verification of the TAU-SIMPACK process chain was conducted by means of generic test cases. Knowing the mass and the moments of inertia of the cargo body allows for exposing it to analytically describable motions. Thus, arbitrary accelerations acting on the body can be preset by manually editing the resulting forces $F$ and moments $M$ in the TAU solution file. Within a given time interval $\Delta t$ these accelerations lead to a displacement of the cargo according to Eqs. (4) and (5). According to figure 6 this modified file is then passed over to SIMPACK. Here, the motion within the time interval $\Delta t$ is discretized with several equally distributed time steps $t^{*}=\Delta t / n$ where $n$ represents the number of sampling points. After every time step $t^{*}$ SIMPACK produces a solution file with information on the current position and attitude of the cargo at this time. This data can be consecutively read into TAU and, after updating the relative position of the respective grids, allows for a visualization of the entire motion. To achieve a successful verification of the process chain the SIMPACK output necessarily has to comply with the analytical solution obtained by employing the basic Eqs. (4) and (5).

$$
\begin{aligned}
\Delta x & =\frac{1}{2} \cdot \frac{F_{x}}{m} \cdot(\Delta t)^{2} \\
\Delta \Theta & =\frac{1}{2} \cdot \frac{M_{y}}{J_{y y}} \cdot(\Delta t)^{2}
\end{aligned}
$$

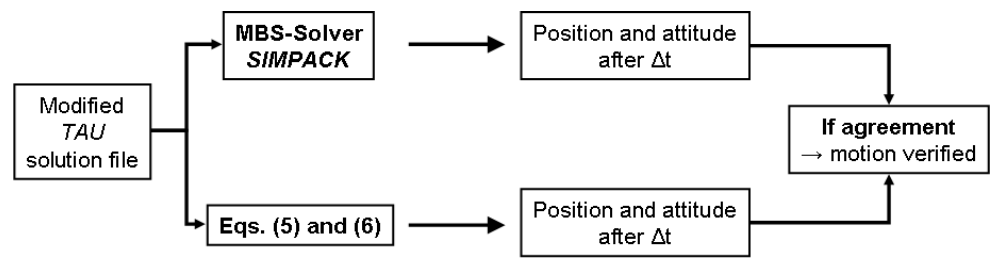

Figure 7. Schematic overview on the verification of the coupled simulation environment.

Figure 7 schematically depicts the aforementioned sequence of the verification process. Figure 8(a) exemplarily shows the visualization of the SIMPACK motion output for each time step $t^{*}$ within the CFD postprocessing environment. As stated above the final position of the cargo has to be in agreement with the analytically determined position and attitude. This procedure was applied for purely translational, purely rotational and combined motions, each under consideration of gravity. The verification of the coupled simulation environment was successfully achieved with this procedure.

\section{III.C.2. Initial Simulation Conditions}

The initial simulation conditions of the cargo were determined by evaluating the experimental trajectories. These are given relative to the characteristic aircraft parameters as summarized in table 2. The initial velocities are related to the wind tunnel onflow velocity. The initial position of the cargo is depicted in figure 8(b). The CFD simulation was carried out with the same onflow conditions as the experiments to allow for a comparability of the results.

Table 2. Relative initial motion parameters of the cargo body at $t=0$ given in the coordinate system as depicted in figure $8(\mathrm{~b})$. The length scales are related to the characteristic length of the aircraft, the angles are related to the aircraft angle of attack. The velocities are related to the onflow velocity.

\begin{tabular}{lc}
\hline \hline Attitude angles $\left(\Phi^{*} / \Theta^{*} / \Psi^{*}\right)$ & $0 / 1.23 / 0$ \\
Angular velocities $(p / q / r)$ & $0 \frac{\mathrm{deg}}{\mathrm{s}} / 199 \frac{\mathrm{deg}}{\mathrm{s}} / 0 \frac{\mathrm{deg}}{\mathrm{s}}$ \\
Translational velocities $\left(v_{x}^{*} / v_{y}^{*} / v_{z}^{*}\right)$ & $-36.1 \cdot 10^{-3} / 0 / 16.7 \cdot 10^{-3}$ \\
\hline \hline
\end{tabular}




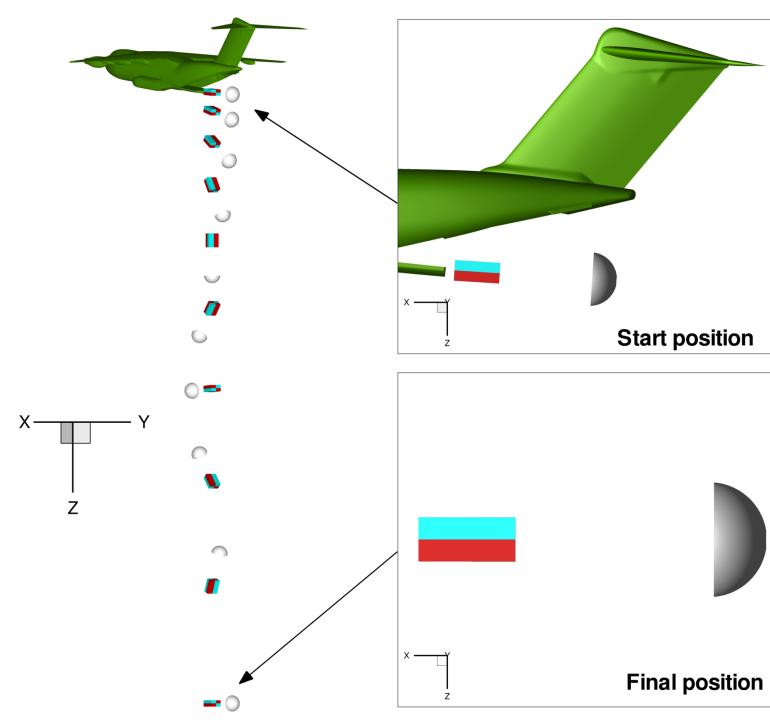

(a) Cargo trajectory during a verification test case.

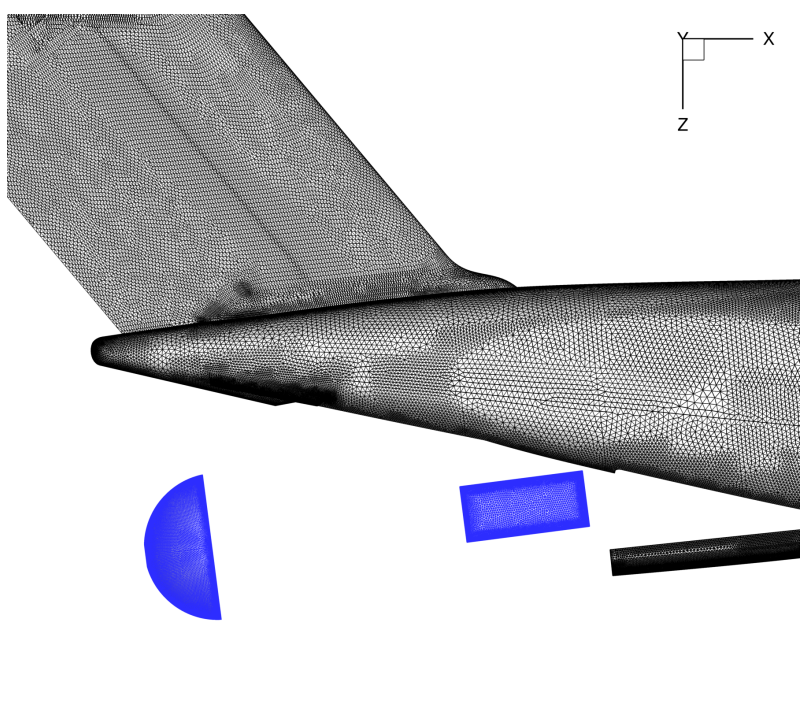

(b) Surface meshes of aircraft and cargo in the initial airdrop position.

Figure 8. Overview on exemplary verification test case and positioning of the bodies at the beginning of the airdrop simulation.

\section{III.C.3. Influence of Unsteady Time Step Size}

As mentioned earlier the simulation was carried out with a sampling rate of $300 \mathrm{~Hz}$, which is equivalent of a time step size of $\Delta t=3.33 \mathrm{~ms}$. To investigate the influence of the unsteady time step size on the simulated trajectory the first $0.1 \mathrm{~s}$ of the trajectory were computed with a sampling rate of $2400 \mathrm{~Hz}(\Delta t=0.417 \mathrm{~ms})$. No differences in position or attitude were found. To save computational resources it was, therefore, decided to run the computation with the larger time step.

\section{III.C.4. Computational Costs}

The computation was carried out in parallel mode on the high-performance $C^{2} A^{2} S^{2} E$ cluster in Braunschweig, using 80 CPUs. The overall wallclock time for the simulation of $0.3 \mathrm{~s}$ of the experimental trajectory totals to $435 \mathrm{~h}$.

\section{Validation of the Numerical Simulation}

For the validation of the numerical simulation the first $0.3 \mathrm{~s}$ of the experimental trajectory were computed. At this time the dropped body has already left the area of influence of the aircraft and is more or less falling balistically without any further changes in attitude. The comparison of the numerical and experimental data is given in figure 9 with the resulting data being depicted in relative size. For that purpose the Euler angles were scaled with the aircraft angle of attack, the velocities with the onflow velocity and the displacement with the characteristic length of the aircraft.

Figure 9(a) depicts the displacement of the centre of gravity of the cargo body relative to its initial position as given in table 2. The latter is also shown in the embedded image with the corresponding coordinate system (positive $x$-direction from tail to nose, positive $z$-direction from aircraft to ground, $y$-axis pointing in starboard direction). It becomes evident that the numerically simulated displacement matches the experimental data very well, both in terms of qualitative and quantitative manner. Regarding the displacement in $y$-direction the experimental data shows a small but increasing offset of the centre of gravity from the symmetry plane with increasing falling distance $z$. The maximum relative displacement amounts to approximately $y^{*}=0.25$. The reason for the latter consists in an asymmetric ejection of the cargo from the aircraft with an initial yaw angle of $\Psi \neq 0^{\circ}$. The numerical simulation, in contrast, shows a symmetric trajectory along the $x z$-plane. 
A comparison of the translational velocities is shown in figure 9(b). Again, the numerical data is in very good agreement with the mean values of the experimental data. The qualitative course of the relative $x$ and $z$-velocities, characterized by a steeper gradient between $t=0.05 \mathrm{~s}$ and $t=0.15 \mathrm{~s}$ and a more shallow gradient from $t=0.15 \mathrm{~s}$ onwards, is well captured by the simulation. The flattening gradient in both cases after $t=0.15 \mathrm{~s}$ can be traced back to a declining intensity of the interference effects between aircraft and cargo. A glance at figure 10 underlines the aforementioned. As for the relative $y$-velocity, the experiments partially disclose an asymmetric motion as discussed in the section above. The simulation shows no velocity component in $y$-direction.

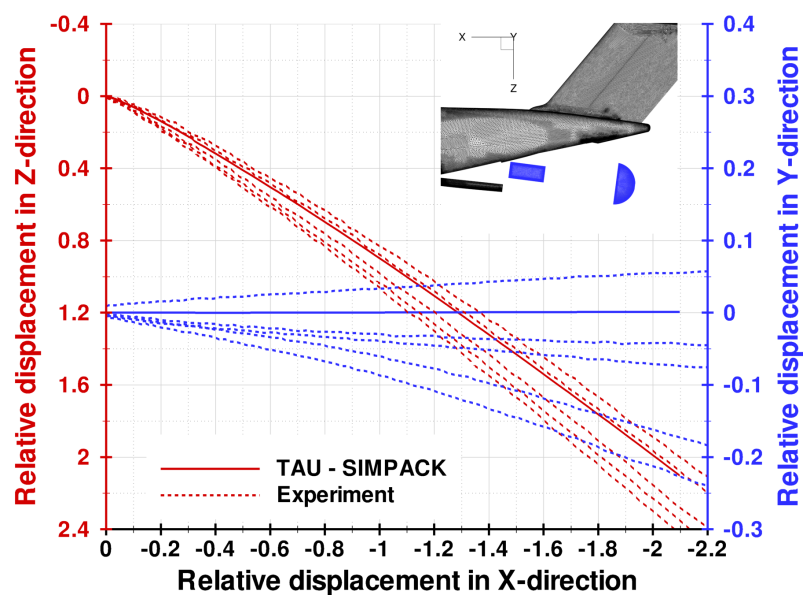

(a) Trajectory of the dropped body.

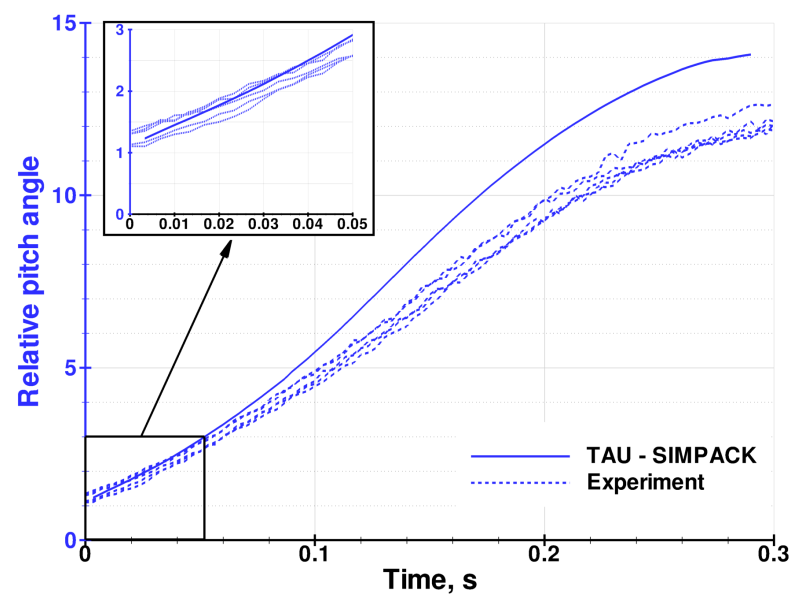

(c) Relative pitch angle of the dropped body.

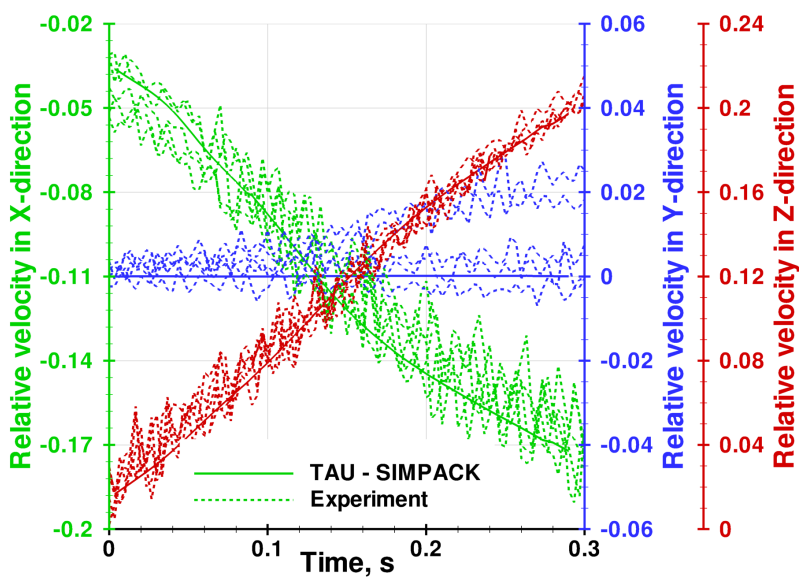

(b) Relative translational velocities.

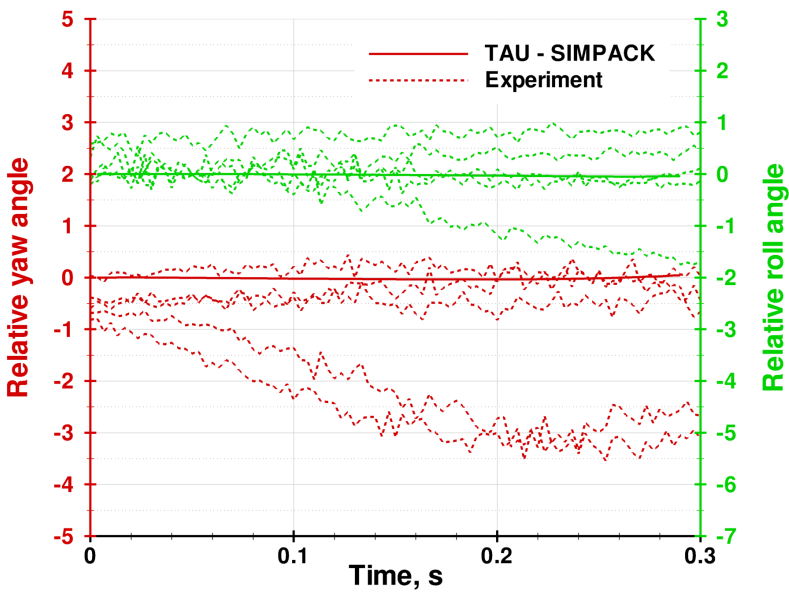

(d) Relative yaw and roll angles of the dropped body.

Figure 9. Comparison of the numerical airdrop simulation with the experimental data.

Figures 9(c) and 9(d) illustrate the attitude of the cargo, using the Euler angle formulation. The qualitative characteristics of the pitch attitude, in particular the changes in curvature, are well-captured by the simulation. In absolute numbers the simulation starts overpredicting the experimental data from $t \approx 0.10 \mathrm{~s}$ onwards. At earlier times, the numerical data complies very well with the experiments as shown in the cut-out in figure $9(\mathrm{c})$. The maximum deviation towards the end of the simulation amounts to $18 \%$. One reason for the differences in pitch angle are probably caused by minor variations in the initial conditions of experiment and simulation. In addition, the variations are probably attributed to disparities between the experimental and numerical surface pressure distributions. An inappropriate resolution of the flow phenomena about the edges of the body, for instance, could explain the deviation in the resulting moments. As no surface pressure data was recorded during experiment a further investigation on this matter is planned in the successor project "MiTraPor II".

The simulation, in contrast, predicts a pure pitching motion of the dropped body without any roll or yaw tendencies. On the contrary the experimental data reveals initial yaw and roll angles of a nonzero value. As 
stated above this is most probably attributed to a tilted position of the cargo in the ejection mechanism. Nevertheless, both the yaw and roll angle nearly remain constant during the fall.

Figure 10 visualizes the trajectory of the dropped body, beginning with the CFD solution after the first time step (figure 10(a)) up to $t=0.25 \mathrm{~s}$. Strong interference effects with the near-field wake of the aircraft can be observed up to $t=0.15 \mathrm{~s}$. Afterwards, the influence of the aircraft diminishes and the increasingly undisturbed flow creates a counter-moment about the pitch axis, preventing the cargo from turning over. As depicted the body is moving with the parachute permanently pointing downwards which results from the chosen experimental setup.

As shown the coupled TAU-SIMPACK environment was successfully validated in the proximate vicinity of the aircraft. Further validation work will be published in $2011 .^{22}$

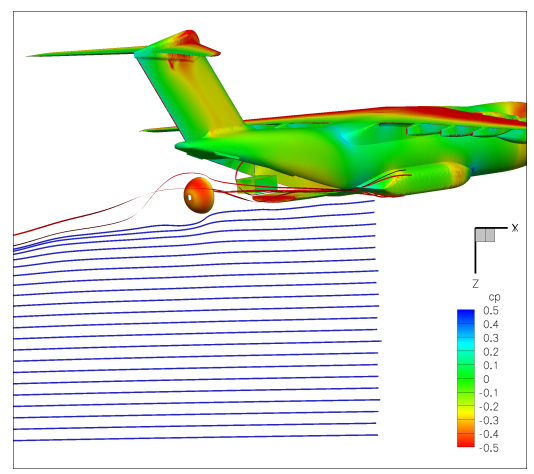

(a) Position after $t=3.33 \mathrm{~ms}$.

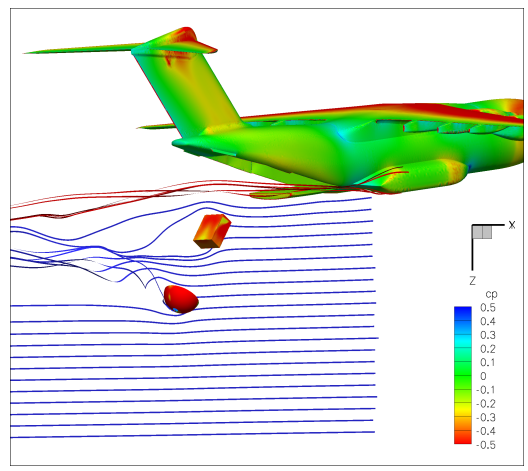

(d) Position after $t=0.15 \mathrm{~s}$.

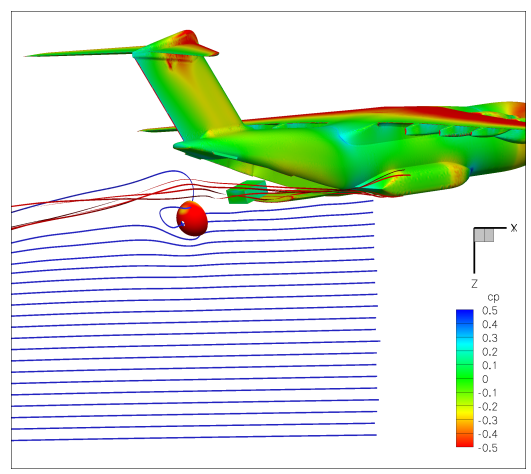

(b) Position after $t=0.05 \mathrm{~s}$

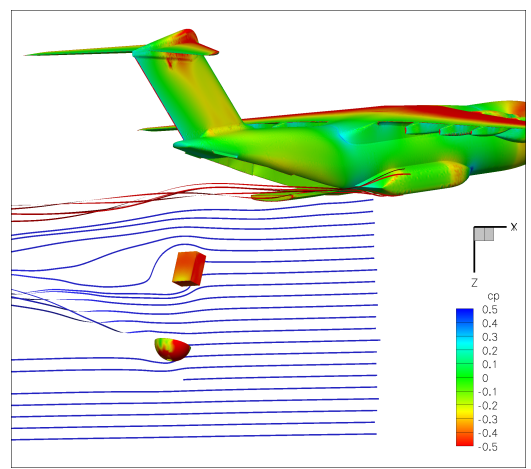

(e) Position after $t=0.20 \mathrm{~s}$.

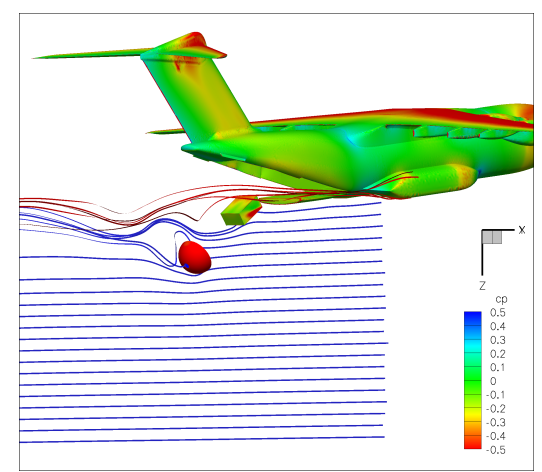

(c) Position after $t=0.10 \mathrm{~s}$.

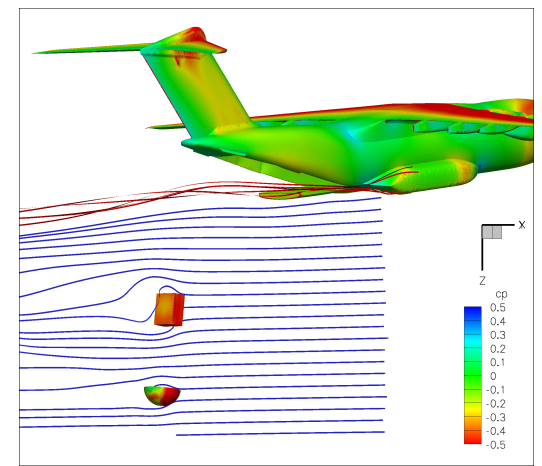

(f) Position after $t=0.25 \mathrm{~s}$.

Figure 10. Trajectory of the dropped supply in the flow field of the aircraft. Surface colouring by pressure coefficient $C_{p}$. Blue streamlines originate on the $x z$-symmetry plane below the aircraft, red streamlines originate near the landing gear fairings.

\section{Conclusion}

The present paper presents selected results of the current DLR activities in developing a well-validated process chain to accurately compute airdrop sequences in the near-field of the aircraft. High-fidelity tools, in particular the DLR TAU code for solving the three-dimensional, unsteady Reynolds-averaged Navier-Stokes equations and the commercial multi-body system software SIMPACK for solving the flight mechanical equations of motion, were brought together in a coupled simulation environment and were verified successfully. Extensive wind tunnel campaigns were carried out to provide validation data for the airdrop simulation. An insight into the wind tunnel scaling requirements, the experimental setup and the measurement instrumentation is given. The developed process chain provides a straightforward, target-orientated approach to support the preparation of real airdrop missions. The numerical airdrop simulation complies very well with the experimental data and is now available for further airdrop investigations.

These are currently been carried out within the DLR project "MiTraPor II". Here, one of the main 
objectives consists in gradually increasing the complexity and degree of realism of the coupled simulation. For instance, the number of moving bodies will be increased. As a first step the trajectory of a cargo body where the rigid parachute is attached by means of a 1-DOF joint will be computed. In a next step, the reaction of the aircraft will be considered during a simulated gravity drop operation. Furthermore, one might think of a more realistic setup of the dropped body itself, for example by placing the cargo onto a tow plate with attached drogue parachute. The parachute model will be enhanced by employing ring slots. The implementation of extraction lines or the consideration of the inflation process might be addressed in future research work.

\section{Acknowledgments}

The work presented in this paper was carried out in the DLR project "MiTraPor - Military Transport Aircraft".

The authors would like to thank A. Raichle, Dr. R. Heinrich and G. Einarsson from the $C^{2} A^{2} S^{2} E$ department of the DLR Institute of Aerodynamics and Flow Technology for their continuous help in establishing the coupled simulation process. Furthermore, the authors like to express their gratitude to the entire team of the DNW-NWB wind tunnel facility in Braunschweig for their very strong support during the test campaigns.

\section{References}

${ }^{1}$ Roosenboom, E. W. M., Schade, N., Acisu, I., and Schröder, A., "Experimental and Numerical Investigation of the Near-Field Wake behind a Transport Aircraft Configuration with an Open Ramp," Paper, $4^{\text {th }}$ Symposium on Integrating CFD and Experiments in Aerodynamics, von Karman Institute, Rhode-Saint-Genèse, Belgium, September 2009.

${ }^{2}$ Roberts, D. W. and Judy, A. D., "Separation Flight Tests of a Small Unmanned Air Vehicle from a C-130 Transport Aircraft," In Flight Test - Sharing Knowledge and Experience, Meeting Proceedings RTO-MP-SCI-162, Paper 19, pp. 19-119-12, Neuilly-sur-Seine, France, 2005.

${ }^{3}$ Benney, R. J., Krainski, W. J., Onckelinx, P., Delwarde, C., Mueller, L., and Vallance, M., "NATO Precision Airdrop Initiatives and Modeling and Simulations Needs," In Fluid Dynamics of Personnel and Equipment Precision Delivery from Military Platforms, Meeting Proceedings RTO-MP-AVT-133, Keynote 2, pp. KN2-1-KN2-22, Neuilly-sur-Seine, France, 2006.

${ }^{4}$ Bagdonovich, B., Desabrais, K. J., Benney, R., Seeger, M., Fossat, F., Vallance, M., and Carlsson, P., "Overview of the Precision Airdrop Improvement Four-Powers Long Term Technology Project," AIAA Paper 2003-2102, 2003.

${ }^{5}$ Seeger, M., Müller, L., Carlsson, P., Bury, Y., de Lassat de Pressigny, Y., Lallemand, G., Vallance, M., Wheeler, R., and Benney, R., "Four-Powers Long Term Technology Projects: 'Airflow Influence on Airdrop' and ' $2^{\text {nd }}$ Precision Airdrop Improvements'," AIAA Paper 2005-1602, 2005.

${ }^{6}$ Cuthbert, P. A., "A Software Simulation of Cargo Drop Tests," AIAA Paper 2003-2132, 2003.

${ }^{7}$ Serrano, M., Leigh, E., III, W. J., Forsythe, J. R., Morton, S. A., and Squires, K. D., "Computational Aerodynamics of the C-130 in Airdrop Configuration," AIAA Paper 2003-0229, 2003.

${ }^{8}$ Desabrais, K. J., "The Motion and Aerodynamics of an Airdrop Platform," AIAA Paper 2004-4845, 2004.

${ }^{9}$ Desabrais, K. J., "Aerodynamic Forces on an Airdrop Platform," AIAA Paper 2005-1634, 2005.

${ }^{10}$ Cavallo, P. A. and Dash, S. M., "Aerodynamics of Multi-Body Separation Using Adaptive Unstructured Grids," AIAA Paper 2000-4407, 2000

${ }^{11}$ Löhner, R. and Baum, J. D., "Three-Dimensional Store Separation Using a Finite Element Solver And Adaptive Remeshing," AIAA Paper 1991-0602, 1991.

${ }^{12}$ Murman, S. M., Aftosmis, M. J., and Berger, M. J., "Simulations of 6-DOF Motion with a Cartesian Method," AIAA Paper 2003-1246, 2003.

${ }^{13}$ Mariscal-Sánchez, F.-J., Cid-Arroyo, S., and Priebe, S., "Computer Simulation of Paratrooper Deployment by Static Line from A400M," In Fluid Dynamics of Personnel and Equipment Precision Delivery from Military Platforms, Meeting Proceedings RTO-MP-AVT-133, Paper 3, pp. 3-1-3-22, Neuilly-sur-Seine, France, 2006.

${ }^{14}$ Sahu, J., Edge, H., Heavey, K., Stein, K., Benney, R., and Chakravarthy, S., "Comparison of Numerical Flow Field Predictions for Army Airdrop Systems," AIAA Paper 1999-1715, 1999.

${ }^{15}$ McQuilling, M. and Potvin, J., "Simulating the Flows About Cargo Containers Used During Parachute Airdrop Operations," AIAA Paper 2010-4565, 2010.

${ }^{16}$ Morton, S. A., Tomaro, R. F., and Noack, R. W., "An Overset Unstructured Grid Methodology Applied to a C-130 with a Cargo Pallet and Extraction Parachute," AIAA Paper 2006-0461, 2006.

${ }^{17}$ Löser, T. and Bergmann, A., "Capabilities of Deployment Tests at DNW-NWB," In Fluid Dynamics of Personnel and Equipment Precision Delivery from Military Platforms, Meeting Proceedings RTO-MP-AVT-133, Paper 13, pp. 13-1-13-11, Neuilly-sur-Seine, France, 2006.

${ }^{18}$ Gerhold, T., "Overview of the Hybrid RANS Code TAU," MEGAFLOW - Numerical Flow Simulation for Aircraft Design, edited by N. Kroll and J. Fassbender, Vol. 89 of Notes on Numerical Fluid Mechanics and Multidisciplinary Design, Springer, 2005, pp. 81-92.

${ }^{19}$ Lugner, P., Arnold, M., and Vaculin, O., "Vehicle System Dynamics," Special issue in memory of Professor Willi Kortüm, Vol. 41, No. 5, $2004,2004$. 
${ }^{20}$ Schindel, L. H., "Store Separation," Agardograph agard-ag-202, 1975.

${ }^{21}$ Schade, N., "Simulation of Store Separation," to appear in: New Results in Numerical and Experimental Fluid Mechanics VIII, edited by A. Dillmann, G. Heller, H.-P. Kreplin, W. Nitsche, and I. Peltzer, Notes on Numerical Fluid Mechanics and Multidisciplinary Design, Springer, 2011.

${ }^{22}$ Schade, N., "Simulations of Trajectories of Cuboid Cargos Released from a Generic Transport Aircraft," AIAA Paper submitted for publication at the $29^{t h}$ AIAA Applied Aerodynamics Conference, Honolulu, USA, 2011.

${ }^{23}$ Bergmann, A., "Modern Wind Tunnel Techniques for Unsteady Testing - Development of Dynamic Testing Rigs," Hermann Schlichting - 100 Years, edited by R. R. et al., Vol. 102 of Notes on Numerical Fluid Mechanics and Multidisciplinary Design, Springer, 2009, pp. 59-77.

${ }^{24}$ F.I.B.U.S, "picColor," http://www.fibus.org/fibusimg.htm, retrieved May 2011.

${ }^{25}$ Gerhold, T., Galle, M., Friedrich, O., and Evans, J., "Calculation of Complex Three-Dimensional Configurations Employing the DLR TAU code," AIAA Paper 1997-0167, 1997.

${ }^{26}$ Schwamborn, D., Gerhold, T., and Heinrich, R., "The DLR TAU-Code: Recent Applications in Research and Industry," In Proceedings of the European Conference on Computational Fluid Dynamics, ECCOMAS, CFD 2006, edited by P. Wesseling, E. Oñate, and J. Périaux, The Netherlands, 2006.

${ }^{27}$ Spalart, P. and Allmaras, S., "A One-Equation Turbulence Model for Aerodynamic Flows," AIAA Paper 1992-439, 1992.

${ }^{28}$ Jameson, A., Schmidt, W., and Turkel, E., "Numerical Solution of the Euler Equations by Finite Volume Methods using Runge-Kutta Time Stepping Schemes," AIAA Paper 81-1259, 1981.

${ }^{29}$ Turkel, E., "Improving The Accuracy of Central Difference Schemes," NASA contractor report 181712, ICASE report no. 88-53, Hampton, Virginia NAS1-18107, 1988.

${ }^{30}$ Turkel, E. and Vatsa, V. N., "Effect of Artificial Viscosity on Three-Dimensional Flow Solutions," AIAA Journal, Vol. 32, No. 1, pp. 39-45, 1994.

${ }^{31}$ Edwards, J. R. and Chandra, S., "Comparison of Eddy-Viscosity Transport Turbulence Models for Three-Dimensional, Shock-Separated Flows," AIAA Journal of Aircraft, Vol. 34, No. 4, pp. 756-763, 1996.

${ }^{32}$ CentaurSoft, "CENTAUR Software," http://www.centaursoft.com, retrieved May 2011.

${ }^{33}$ Madrane, A., Heinrich, R., and Gerhold, T., "Implementation of the Chimera Method in the Unstructured Hybrid DLR Finite Volume TAU-Code," Paper, $6^{\text {th }}$ Overset Composite Grid and Solution Technology Symposium, Ft. Walton Beach, Florida, USA, October 2002.

${ }^{34}$ Madrane, A., Raichle, A., and Stürmer, A., "Parallel Implementation of a Dynamic Overset Unstructured Grid Approach," Proceedings of the Third International Conference on Computational Fluid Dynamics, ICCFD3, European Community on Computational Methods in Applied Sciences, Regensburg, Germany, 2004.

${ }^{35}$ Schütte, A., Einarsson, G., Raichle, A., Schöning, B., Mönnich, W., Orlt, M., Neumann, J., Arnold, J., and Forkert, T., "Numerical Simulation of Maneuvring Aircraft by Aerodynamic, Flight-Mechanics, and Structural-Mechanics Coupling," AIAA Journal of Aircraft, Vol. 46, No. 1, pp. 53-64, 2009.

${ }^{36}$ Spalart, P. R., Deck, S., Shur, M. L., Squires, K. D., Strelets, M. K., and Travin, A., "A New Version of Detached-eddy Simulation, Resistant to Ambiguous Grid Densities," Theoretical and Computational Fluid Dynamics, Vol. 20, No. 3, pp. 181-195, Springer, 2006.

${ }^{37}$ Krüger, W., Heinrich, R., and Spieck, M., "Fluid-Structure Coupling Using CFD and Multibody Simulation Methods," Proceedings, International Congress of The Aeronautical Sciences (ICAS), Toronto, Canada, 2002.

${ }^{38}$ Phillips, W. F., Hailey, C. E., and Gebert, G. A., "Review of Attitude Representations Used for Aircraft Kinematics," AIAA Journal of Aircraft, Vol. 38, No. 4, pp. 718-737, 2001.

${ }^{39}$ Arnold, J. and Einarsson, G., "Multibody Simulation of a Generic Delta Wing Configuration in Roll Motion," Paper 2007-116, International Forum on Aeroelasticity and Structural Dynamics (IFASD), June 2007. 